\title{
Clinical Factors Associated With Dose Escalation of Solifenacin for the Treatment of Overactive Bladder in Real Life Practice
}

\author{
Ji-Youn Chun, Miho Song, Ji-Yeon Han ${ }^{1}$, Selee Na², Bumsik Hong, Myung-Soo Choo \\ Department of Urology, Asan Medical Center, University of Ulsan College of Medicine, Seoul; \\ ${ }^{1}$ Department of Urology, Pusan National University Yangsan Hospital, Pusan National University School of Medicine, Yangsan; \\ ${ }^{2}$ Department of Computer Science and Information Systems, Hanyang Women's University, Seoul, Korea
}

\begin{abstract}
Purpose: To determine the baseline clinical characteristics associated with dose escalation of solifenacin in patients with overactive bladder $(\mathrm{OAB})$.

Methods: We analyzed the data of patients with $\mathrm{OAB}$ (micturition frequency $\geq 8 /$ day and urgency $\geq 1$ /day) who were treated with solifenacin and followed up for 24 weeks. According to our department protocol, all the patients kept voiding diaries, and $\mathrm{OAB}$ symptom scores (OABSS) were monitored at baseline and after 4, 12, and 24 weeks of solifenacin treatment.

Results: In total, 68 patients (mean age, $60.8 \pm 10.0$ years) were recruited. The dose escalation rate by the end of the study was $41.2 \%$, from $23.5 \%$ at 4 weeks and $17.6 \%$ at 12 weeks. At baseline, the dose escalator group had significantly more OAB wet patients $(53.6 \%$ vs. $20.0 \%)$ and higher total OABSS $(10.2 \pm 2.4$ vs. $7.9 \pm 3.5, \mathrm{P}=0.032)$ than the nonescalator group. OAB wet (odds ratio [OR], 4.615; 95\% confidence interval [CI], 1.578-13.499; $\mathrm{P}<0.05)$ and total OABSS (OR, 1.398; 95\% CI, 1.046-1.869; $\mathrm{P}<0.05)$ were found to be independently associated with dose escalation.

Conclusions: Patients who have urgency urinary incontinence and high total OABSS have a tendency for dose escalation of solifenacin.
\end{abstract}

Keywords: Overactive urinary bladder; Muscarinic antagonists; Solifenacin

\section{INTRODUCTION}

The International Continence Society defines overactive bladder $(\mathrm{OAB})$ as urgency with or without urgency urinary incontinence (UUI), usually accompanied by daytime frequency and nocturia, in the absence of urinary tract infection (UTI) or other obvious pathology [1]. The overall prevalence of lower urinary tract symptoms was $61.4 \%$ (53.7\% of men, $68.9 \%$ of women) in the Korean EPIC study [2] and $11.8 \%$ (10.8\% of men, $12.8 \%$ of women) in the international EPIC study [3], and the prevalence of $\mathrm{OAB}$ increased with age. $\mathrm{OAB}$ has a marked influence on quality of life. Liberman et al. [4] reported that individuals with $\mathrm{OAB}$ experienced decreased quality of life relative to community controls, even if they did not have incontinence.
Current treatment options include lifestyle modifications, behavioral therapy, pharmacotherapy, intravesical injection of botulinum toxin, and surgical procedures such as bladder augmentation or sacral neuromodulation. However, antimuscarinic agents represent the mainstay of OAB treatment. Flexible dosing of anticholinergic drugs is useful in clinical practice for achieving the maximal effect with minimal side effects [5].

Data from numerous flexible-dose studies of solifenacin demonstrated significantly improved urgency, frequency, and nocturia symptoms and increased volume voided in OAB dry patients [6-9]. However, these clinical trials were conducted with $\mathrm{OAB}$ patients for 14 weeks and offered only one opportunity for dose escalation.

The aim of the present study was to determine the baseline
Corresponding author: Myung-Soo Choo

Department of Urology, Asan Medical Center, 88 Olympic-ro 43-gil, Songpa-gu, Seoul 138-736, Korea

Tel: +82-2-3010-3735 / Fax: +82-2-477-8928 / E-mail: mschoo@amc.seoul.kr Submitted: January 5, 2014 / Accepted after revision: March 4, 2014
This is an Open Access article distributed under the terms of the Creative Commons Attribution Non-Commercial License (http://creativecommons.org/licenses/by-nc/3.0/) which permits unrestricted non-commercial use, distribution, and reproduction in any medium, provided the original work is properly cited. 
clinical characteristics associated with solifenacin DE in practice. In addition, we assessed the efficacy of solifenacin DE in reducing OAB symptoms after DE. We provided two opportunities for DE (after 4 and 12 weeks), and the patients were followed up for 24 weeks.

\section{MATERIALS AND METHODS}

This was a retrospective investigation of the factors associated with solifenacin DE in a population with $\mathrm{OAB}$ symptoms according to the protocol of our hospital. We reviewed 2,332 patients with $\mathrm{OAB}$ who received 5-mg solifenacin between August 2010 and January 2012. Men and women aged $\geq 20$ years with a mean micturition frequency $\geq 8$ /day, urgency episodes $\geq 1$ /day in a 3-day voiding diary at baseline, and follow-up of at least 24 weeks were selected. Patients were excluded if they were taking alpha blockers or 5-alpha reductase inhibitors or had done so in the previous 2 months, had undergone pelvic surgery within 1 year (hysterectomy, sling operation, etc.), had received previous $\mathrm{OAB}$ treatment with antimuscarinics within 3 months, or had specific comorbidities such as known neurogenic bladder, history of acute urinary retention requiring catheterization, predominant stress urinary incontinence, significant pelvic organ prolapse, or lower urinary tract surgery within 6 months.

According to our department protocol, all the patients kept 3-day voiding diaries, and OAB symptom scores (OABSS) [10] were monitored at baseline and after 4,12 , and 24 weeks of solifenacin treatment. The subjects recorded the time of every micturition and rated the sensation associated with each using the 5-point urinary sensation scale (USS) [11].

Urgency grade was defined as the mean of urgency episodes of USS ratings for all micturitions reported during the 3-day voiding diary period. All the patients received 5-mg solifenacin once daily before sleep for 4 weeks. After 4 weeks, the subjects either maintained the solifenacin dosage at $5 \mathrm{mg}$ once daily (nonescalators) or increased their dose to $10 \mathrm{mg}$ once daily for the remainder of the study (escalators), based on discussions between the subjects and the investigator regarding their subjective assessment of treatment efficacy and tolerability. Similarly, after 12 weeks, the patients either remained at the baseline dose or increased it for the remaining 12 weeks of the study (escalators) after consultation with the investigator regarding efficacy and tolerability. This study did not need an institutional review board approval because all the patients were followed up according to the protocol of our hospital, the data were collect- ed by retrospective chart reviews of existing medical records, and the data were recorded by the investigator anonymously to protect the patients' identities.

The patients were divided according to "no escalation" (NE) or "dose escalation" (DE). The DE group consisted of the patients who elevated their solifenacin dose from 5 to $10 \mathrm{mg}$ at either week 4 or 12.

Patients were considered to have OAB-Wet if they had $\mathrm{OAB}$ symptoms of urgency and frequency with urge urinary incontinence. OAB-Dry was defined as patients with symptoms of urgency and frequency but lacked urge urinary incontinence.

Changes in $\mathrm{OAB}$ symptoms were evaluated from baseline to 4,12 , and 24 weeks for both groups. The baseline clinical factors associated with $\mathrm{DE}$ were assessed using a logistic regression model. The baseline demographic characteristics of the NE and DE groups were evaluated for statistical differences using the analysis of variance or $\mathrm{t}$-tests for continuous variables and chisquare tests for categorical variables. The baseline clinical risk factors for DE over the entire study period and at 4 and 12 weeks were examined by univariate and multivariate analyses using a logistic regression model. Both absolute (numeric mean) and relative (\%) changes in efficacy variables were assessed because of the potential for baseline differences in OAB symptom severity between the NE and DE groups.

Least-squares (LS) mean differences in changes from baseline in the $\mathrm{DE}$ and $\mathrm{NE}$ groups were assessed using a linear mixed model. Relative percent changes in efficacy variables from baseline to 4 and 12 weeks within each DE group were assessed using a Wilcoxon signed-rank test at the $5 \%$ significance level. Relative differences in mean percent changes between the NE and DE groups were assessed by two-sided paired t-tests. All the reported $\mathrm{P}$-values were two-sided, and $\mathrm{P}<0.05$ was deemed statistically significant. Data manipulation and statistical analyses were conducted using SAS ver. 9.2 (SAS Institute Inc., Cary, NC, USA).

\section{RESULTS}

\section{Subjects}

In total, 68 patients (54 women and 14 men; mean age, $60.8 \pm$ 10.0 years) were selected. The NE and DE groups were comparable with respect to baseline demographics (Table 1). Thirty six percent of male patients (4/11) were taking alpha-blockers over 2 months at the beginning of the medication with solifenacin. The one of the exclusion criteria was taking alpha-blocker 
Table 1. Baseline demographic characteristics of the nonescalators and escalators group

\begin{tabular}{|c|c|c|c|}
\hline Parameter & Nonescalators & Escalators & P-value \\
\hline Sex & & & 0.082 \\
\hline Male & $11(16.2)$ & $3(4.4)$ & \\
\hline Female & $29(42.6)$ & $25(36.8)$ & \\
\hline Age (yr) & $61.9 \pm 9.4$ & $59.9 \pm 10.5$ & 0.430 \\
\hline Body mass index $\left(\mathrm{kg} / \mathrm{m}^{2}\right)$ & $24.8 \pm 3.5$ & $23.8 \pm 2.5$ & 0.316 \\
\hline OAB type & & & $0.004^{\mathrm{a})}$ \\
\hline OAB dry & $32(47.0)$ & $13(19.1)$ & \\
\hline OAB wet & $8(11.8)$ & $15(22.1)$ & \\
\hline \multicolumn{4}{|l|}{ Diary variables per $24 \mathrm{hr}$} \\
\hline Micturitions & $10.7 \pm 4.0$ & $11.5 \pm 3.2$ & 0.204 \\
\hline Urgency episodes & $6.2 \pm 5.0$ & $8.4 \pm 4.4$ & 0.055 \\
\hline UUI episodes & $0.4 \pm 1.2$ & $1.1 \pm 2.1$ & 0.079 \\
\hline Urgency grade & $3.2 \pm 0.3$ & $3.4 \pm 0.9$ & 0.055 \\
\hline \multicolumn{4}{|l|}{ Questionnaire scores } \\
\hline OABSS & $7.9 \pm 3.5$ & $10.2 \pm 2.4$ & $0.032^{\mathrm{a})}$ \\
\hline OABSS-3Q & $3.0 \pm 1.2$ & $3.8 \pm 1.1$ & 0.048 \\
\hline \multicolumn{4}{|l|}{ Uroflowmetry } \\
\hline Voiding volume (mL) & $256.5 \pm 115.8$ & $219.8 \pm 110.8$ & 0.196 \\
\hline Peak flow rate $(\mathrm{mL} / \mathrm{sec})$ & $22.6 \pm 9.2$ & $23.2 \pm 11.4$ & 0.806 \\
\hline $\begin{array}{l}\text { Postvoided residual } \\
\text { urine }(\mathrm{mL})\end{array}$ & $27.4 \pm 38.7$ & $23.3 \pm 45.5$ & 0.693 \\
\hline
\end{tabular}

Values are presented as number (\%) or mean \pm standard deviation. $\mathrm{OAB}$, overactive bladder; UUI, urgency urinary incontinence; OABSS, OAB symptom scores.

${ }^{\text {a) }} \mathrm{P}<0.05$.

within 2 months, so this 4 patients were included to present study finally. No other patients add with alpha-blockers or 5-alpha reductase inhibitor during the study except this 4 patients.

The rate of DE from the starting dose of 5-mg solifenacin to 10-mg solifenacin over the entire treatment period was $41.2 \%$ (28/68), from $23.5 \%(16 / 68)$ at 4 weeks and $17.6 \%(12 / 68)$ at 12 weeks. After 12 weeks, however, one patient reduced the dose until the end of treatment because of an adverse effect (dry mouth).

The DE group shows higher mean values for baseline micturitions (11.5 $\pm 3.2 / 24$ hours vs. $10.7 \pm 4.0 / 24$ hours, $\mathrm{P}=0.204)$, urgency episodes ( $8.4 \pm 4.4 / 24$ hours vs. $6.2 \pm 5.0 / 24$ hours, $\mathrm{P}=0.055)$, UUI episodes $(1.1 \pm 2.1 / 24$ hours vs. $0.4 \pm 1.2 / 24$ hours, $\mathrm{P}=0.079)$, and urgency grade $(3.4 \pm 0.9 / 24$ hours vs. $3.2 \pm 0.3 / 24$ hours, $\mathrm{P}=0.055)$ than the NE group, even though it was not statistically significant. This results showed that the $\mathrm{DE}$ group had more severe objective OAB symptoms than NE group. Compared with the NE group, the DE group also showed significantly higher baseline OABSS $(7.9 \pm 3.5$ vs. $10.2 \pm 2.4, \mathrm{P}=0.032)$ and OABSS-3Q $(3.0 \pm 1.2$ vs. $3.8 \pm 1.1$, $\mathrm{P}=0.048)$. Baseline voiding parameters such as voiding volume, maximal urinary flow rate, and postvoided residual urine were generally similar in both groups (all $\mathrm{P}>0.05$ ).

\section{Factors Associated With DE}

The univariate analysis indicated that age, sex, body mass index, and voiding parameters did not influence the $\mathrm{DE}$ outcome in the DE group (Table 2). Patients with OAB wet (odds ratio [OR], 4.615; 95\% confidence interval [CI], 1.578-13.499; $\mathrm{P}=0.002$ ) and higher total OABSS (OR, 1.398; 95\% CI, 1.046-1.869; P< 0.05 ) were more likely to escalate the dose than patients with $\mathrm{OAB}$ dry in the total DE group. The number of urgency and UUI episodes per 24 hours were marginally associated with DE in the total DE group (OR, 1.109; 95\% CI, 0.998-1.224; $\mathrm{P}=0.058$ and $\mathrm{OR}, 1.704 ; 95 \% \mathrm{CI}, 0.977-2.975 ; \mathrm{P}=0.073$ ). The multivariate analysis indicated that no factor was independently associated with solifenacin DE. UUI episodes and OABSS-Q3 were excluded from the multivariate analysis because these factors were related to multicollinearity of risk factors.

In the 4-week escalators (4WE), OAB wet and voiding volume were associated with $\mathrm{DE}$ in the subgroup univariate analysis (OR, 9.351; 95\% CI, 1.092-19.422; $\mathrm{P}=0.039$ and OR, 0.994; 95\% CI, 0.988-1.000; $\mathrm{P}=0.045$ ) (Table 3). The number of urgency episodes and total OABSS per 24 hours were marginally associated in the $4 \mathrm{WE}$ (OR, 1.188; 95\% CI, 0.996-1.254; $\mathrm{P}=$ 0.058 and $\mathrm{OR}, 1.289 ; 95 \% \mathrm{CI}, 0.954-1.741 ; \mathrm{P}=0.098)$. In the 12week escalators, only urgency grade was associated with $\mathrm{DE}$ (OR, 4.060; 95\% CI, 1.126-14.630; $\mathrm{P}=0.032$ ) in the subgroup univariate analysis.

\section{Efficacy}

After 4 weeks, significant percentage improvements in mean micturitions, urgency episodes, and UUI episodes were observed from the baseline in both groups (Table 4). In the NE group, micturitions (50.9\%), urgency episodes (85.4\%), and UUI episodes $(77.0 \%)$ decreased from the baseline (all $\mathrm{P}<0.05$ ). In the DE group, micturitions (25.5\%), urgency episodes (57.4\%), and UUI episodes (18.8\%) decreased from the baseline (all $\mathrm{P}<0.05$ ). The mean percentage change in the NE group was significantly higher than that in the DE group (all $\mathrm{P}<0.05$ ). When mean changes were assessed, all the diary variables per 
Table 2. Factors related to solifenacin dose escalation in the total dose escalators

\begin{tabular}{|c|c|c|c|c|c|c|}
\hline \multirow{2}{*}{ Variable } & \multicolumn{3}{|c|}{ Univariate analysis } & \multicolumn{3}{|c|}{ Multivariate analysis } \\
\hline & OR & $95 \% \mathrm{CI}$ & P-value & OR & $95 \% \mathrm{CI}$ & P-value \\
\hline Sex (female) & 0.334 & $0.170-1.825$ & 0.334 & & & \\
\hline Age & 0.980 & $0.933-1.030$ & 0.424 & & & \\
\hline Body mass index & 0.899 & $0.761-1.105$ & 0.899 & & & \\
\hline \multicolumn{7}{|l|}{ OAB type } \\
\hline OAB wet & 4.615 & $1.578-13.499$ & $0.002^{\mathrm{a})}$ & 1.234 & $0.916-1.662$ & 0.167 \\
\hline \multicolumn{7}{|l|}{ Diary variables per $24 \mathrm{hr}$} \\
\hline Micturitions & 1.101 & $0.946-1.281$ & 0.213 & & & \\
\hline Urgency episodes & 1.109 & $0.988-1.244$ & 0.058 & & & \\
\hline UUI episodes & 1.704 & $0.977-2.975$ & 0.073 & & & \\
\hline Urgency grade & 0.569 & $0.218-1.488$ & 0.251 & & & \\
\hline \multicolumn{7}{|l|}{ Questionnaire scores } \\
\hline OABSS & 1.398 & $1.046-1.869$ & $0.024^{\mathrm{a})}$ & 0.784 & $0.572-1.074$ & 0.125 \\
\hline OABSS-3Q & 1.853 & $0.989-3.475$ & 0.172 & & & \\
\hline \multicolumn{7}{|l|}{ Uroflowmetry } \\
\hline Voiding volume & 0.997 & $0.993-1.002$ & 0.196 & & & \\
\hline Peak flow rate & 1.003 & $0.960-1.055$ & 0.802 & & & \\
\hline Postvoided residual urine & 0.996 & $0.986-1.009$ & 0.688 & & & \\
\hline
\end{tabular}

$\mathrm{OR}$, odds ratio; $\mathrm{CI}$, confidence interval; $\mathrm{OAB}$, overactive bladder; $\mathrm{UUI}$, urgency urinary incontinence; $\mathrm{OABSS}$, OAB symptom scores. ${ }^{a)} \mathrm{P}<0.05$.

24-hour period in the NE group were significantly greater than those in the DE group (Fig. 1). The mean numbers of micturitions ( -2.94 vs. -0.87 ; LS mean difference, 2.07 ; $\mathrm{P}>0.05$ ), urgency episodes ( -4.53 vs. -3.64 ; LS mean difference, 0.89 ; $\mathrm{P}>0.05$ ), UUI episodes ( -0.41 vs. 0.09 ; $\mathrm{LS}$ mean difference, 0.50 ; $\mathrm{P}>0.05)$, and urgency grade ( -1.57 vs. -0.43 ; LS mean difference, 1.14; $\mathrm{P}>0.05)$ per 24 hours in the NE group were higher than those in the DE group (Fig. 1).

After 12 weeks, significant mean percentage improvements were observed in all the voiding diary variables compared with the baseline in both groups (Table 4). In the NE group, micturitions (18.9\%), urgency episodes (85.4\%), and UUI episodes (84.6\%) decreased from the baseline (all $\mathrm{P}<0.05$ ), as well as in the $\mathrm{DE}$ group at $22.4 \%, 75.0 \%$, and $59.8 \%$, respectively (all $\mathrm{P}<0.05$ ). When the mean percentage change was assessed, the improvement in UUI episodes per 24 hours in the NE group was significantly greater than that in the $\mathrm{DE}$ group after 12 weeks $(\mathrm{P}=0.034)$.

The $\mathrm{DE}$ group showed an improvement in micturitions and urgency episodes per 24 hours, as did the NE group $(\mathrm{P}=0.831$ and $\mathrm{P}=0.466$ ). In contrast to the week 4 results, the mean numbers of micturitions ( -3.13 vs. -3.15 ; LS mean difference, 0.02 ;
$\mathrm{P}>0.05)$, urgency episodes ( -4.67 vs. -5.45 ; LS mean difference, 0.78 ; $\mathrm{P}>0.05$ ), and UUI episodes ( -0.44 vs. -0.53 ; LS mean difference, 0.09 ; $\mathrm{P}>0.05)$ per 24 hours, showed similar or greater reductions in value in the $\mathrm{DE}$ group compared with the $\mathrm{NE}$ group except urgency grade (Fig. 1).

After 24 weeks, significant mean percentage improvements were observed from the baseline in all the voiding diary variables in both groups (Table 4). In the NE group, micturitions (64.7\%), urgency episodes (96.3\%), and UUI episodes (92.3\%) decreased from the baseline (all $\mathrm{P}<0.05$ ), as well as in the DE group at $62.6 \%, 79.6 \%$, and $75.6 \%$, respectively (all $\mathrm{P}<0.05$ ). No significant differences in mean percentage changes in the diary variables were observed between the two groups (all $\mathrm{P}>0.05$ ). Following $\mathrm{DE}$, the drug efficacy was continuous and improved in both groups. The mean numbers of micturitions ( -3.49 vs. -3.44 ; LS mean difference, $0.05 ; \mathrm{P}>0.05)$, urgency episodes $(-4.92$ vs. -5.97 ; LS mean difference, $1.05 ; \mathrm{P}>0.05$ ), and UUI episodes ( -0.38 vs. -1.32 ; LS mean difference, $0.94 ; \mathrm{P}>0.05)$ per 24 hours were greater in the $\mathrm{DE}$ group compared with the NE group. Only the urgency grade $(-2.43$ vs. -1.53 ; LS mean difference, $0.90 ; \mathrm{P}>0.05)$ per 24 hours was lower in the DE group compared with the NE group (Fig. 1). 
Table 3. Univariate analysis of the factors related to solifenacin dose escalation in the 4-, and 12-week dose escalators

\begin{tabular}{|c|c|c|c|c|c|c|}
\hline \multirow{2}{*}{ Variable } & \multicolumn{3}{|c|}{4 Weeks } & \multicolumn{3}{|c|}{12 Weeks } \\
\hline & OR & $95 \% \mathrm{CI}$ & $\mathrm{P}$-value & OR & $95 \% \mathrm{CI}$ & P-value \\
\hline \multicolumn{7}{|l|}{ Sex (female) } \\
\hline Age & 2.100 & $0.417-10.569$ & 0.368 & 4.550 & $0.544-38.023$ & 0.162 \\
\hline Body mass index & 0.990 & $0.936-1.048$ & 0.728 & 0.964 & $0.909-1.023$ & 0.229 \\
\hline OAB type & 1.137 & $0.849-1.524$ & 0.389 & 0.842 & $0.654-1.084$ & 0.183 \\
\hline OAB wet & 9.351 & $1.092-19.422$ & $0.039^{\mathrm{a})}$ & 4.245 & $0.646-8.143$ & 0.199 \\
\hline \multicolumn{7}{|l|}{ Diary variables per $24 \mathrm{hr}$} \\
\hline Micturitions & 1.131 & $0.977-1.310$ & 0.100 & 0.906 & $0.739-1.109$ & 0.339 \\
\hline Urgency episodes & 1.188 & $0.996-1.254$ & 0.058 & 1.004 & $0.892-1.130$ & 0.947 \\
\hline UUI episodes & 1.112 & $0.806-1.534$ & 0.519 & 1.593 & $0.916-1.754$ & 0.153 \\
\hline Urgency grade & 0.654 & $0.256-1.674$ & 0.376 & 4.060 & $1.126-14.630$ & $0.032^{\mathrm{a})}$ \\
\hline \multicolumn{7}{|l|}{ Questionnaire scores } \\
\hline OABSS & 1.289 & $0.954-1.741$ & 0.098 & 1.237 & $0.932-1.724$ & 0.131 \\
\hline OABSS-3Q & 1.502 & $0.759-2.974$ & 0.243 & 1.413 & $0.675-2.957$ & 0.359 \\
\hline \multicolumn{7}{|l|}{ Uroflowmetry } \\
\hline Voiding volume & 0.994 & $0.988-1.000$ & 0.045 & 1.000 & $0.994-1.005$ & 0.866 \\
\hline Peak flow rate & 0.983 & $0.929-1.041$ & 0.555 & 1.019 & $0.965-1.077$ & 0.498 \\
\hline Post-voided residual urine & 1.003 & $0.990-1.013$ & 0.650 & 0.987 & $0.966-1.007$ & 0.197 \\
\hline
\end{tabular}

OR, odds ratio; $\mathrm{CI}$, confidence interval; $\mathrm{OAB}$, overactive bladder; UUI, urgency urinary incontinence; OABSS, OAB symptom scores. ${ }^{\text {a) } \mathrm{P}}<0.05$.

Table 4. Mean percentage changes in efficacy parameters from baseline for the nonescalators and escalators (Mann-Whitney test)

\begin{tabular}{|c|c|c|c|c|c|c|c|c|c|}
\hline \multirow{2}{*}{$\begin{array}{l}\text { Diary variables } \\
\text { per } 24 \mathrm{hr}\end{array}$} & \multicolumn{3}{|c|}{4 Weeks } & \multicolumn{3}{|c|}{12 Weeks } & \multicolumn{3}{|c|}{24 Weeks } \\
\hline & $\begin{array}{l}\text { Nonescala- } \\
\text { tors }\end{array}$ & $\begin{array}{c}\text { Dose } \\
\text { escalators }\end{array}$ & $\mathrm{P}$-value & $\begin{array}{l}\text { Nonescala- } \\
\text { tors }\end{array}$ & $\begin{array}{c}\text { Dose } \\
\text { escalators }\end{array}$ & $\mathrm{P}$-value & $\begin{array}{l}\text { Nonescala- } \\
\text { tors }\end{array}$ & $\begin{array}{c}\text { Dose } \\
\text { escalators }\end{array}$ & P-value \\
\hline Micturitions & $-50.9^{\mathrm{a})}$ & $-25.5^{\mathrm{a})}$ & 0.007 & $-18.9^{\mathrm{a})}$ & $-22.4^{\mathrm{a})}$ & 0.831 & $-64.7^{\mathrm{a})}$ & $-62.6^{a)}$ & 0.826 \\
\hline Urgency episodes & $-85.4^{\mathrm{a})}$ & $-57.4^{\mathrm{a})}$ & 0.002 & $-85.4^{\mathrm{a})}$ & $-75.0^{\mathrm{a})}$ & 0.466 & $-96.3^{\mathrm{a})}$ & $-79.6^{\mathrm{a})}$ & 0.357 \\
\hline UUI episodes & $-77.0^{\mathrm{a})}$ & $-18.8^{\mathrm{a})}$ & 0.039 & $-84.6^{\mathrm{a})}$ & $-59.8^{\mathrm{a})}$ & 0.034 & $-92.3^{\mathrm{a})}$ & $-75.6^{\mathrm{a})}$ & 0.239 \\
\hline
\end{tabular}

For the UUI episodes, only the patients with UUI episode at baseline characteristics were analyzed.

UUI, urgency urinary incontinence.

${ }^{\text {a) }} \mathrm{P}<0.05$ versus the baseline (Wilcoxon signed rank test).

\section{DISCUSSION}

Of the patients' baseline characteristics, the values for $\mathrm{OAB}$ wet, all voiding diary variables, and total OABSS in the 10-mg solifenacin group were greater than those in the 5 -mg solifenacin group. The key factors associated with $\mathrm{DE}$ were $\mathrm{OAB}$ wet and total OABSS across all DE groups. All diary variables decreased from baseline to 12 and 24 weeks throughout the study period in both groups.

Antimuscarinic drugs, which have proven efficacy in patients who have $\mathrm{OAB}$ symptoms with or without urodynamically confirmed detrusor overactivity, were used as the first-line treatment [12]. Some agents are available in two or more dose strengths; a flexible dosing strategy can help to achieve the desired efficacy with minimal side effects [13].

Several studies have evaluated baseline characteristics associated with $\mathrm{DE}$ in $\mathrm{OAB}$ patients. Most studies of solifenacin flexible-dose strategies were performed using a 12- to 16-week study period $[6,8,9]$. After 4 or 8 weeks of treatment, the patients had a single opportunity either to continue with their original dose 


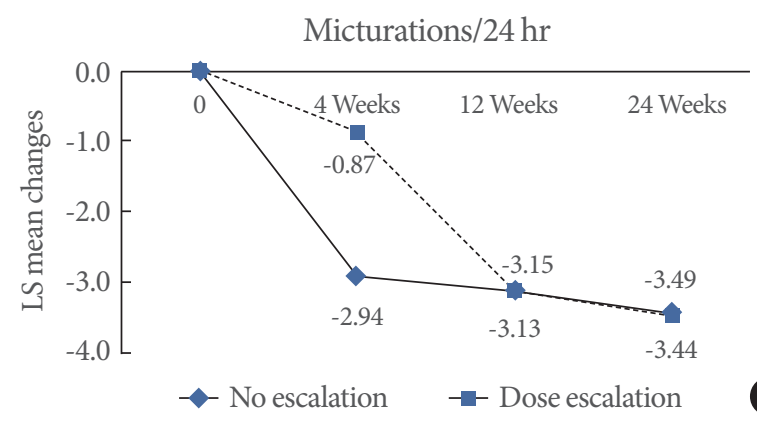

UUI episodes $/ 24 \mathrm{hr}$

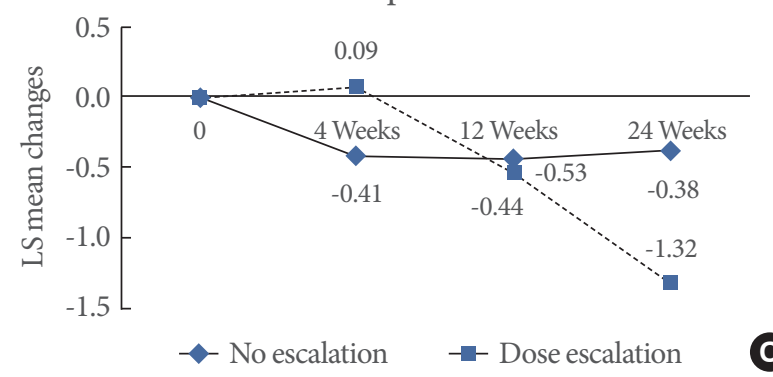

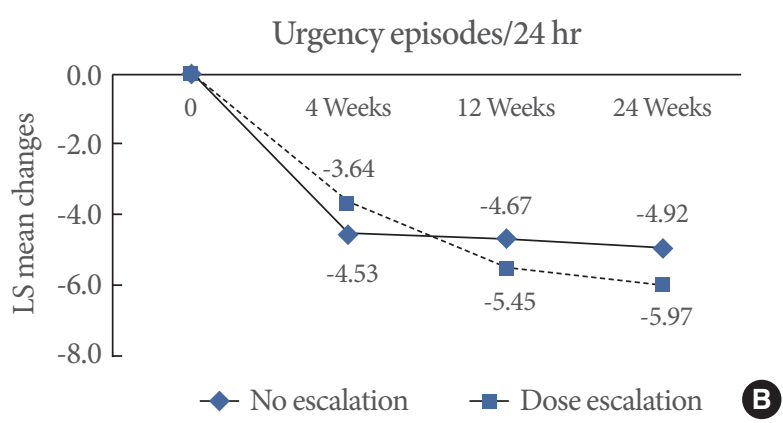

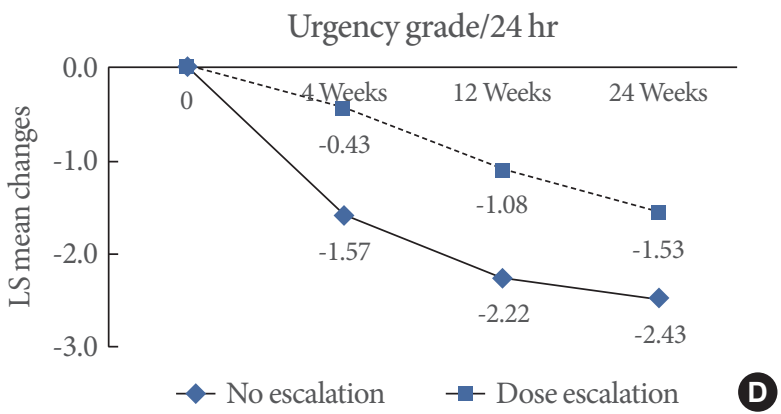

Fig. 1. Least-squares (LS) mean changes from baseline in number of (A) micturitions, (B) urgency episodes, (C) UUI episodes, and (D) urgency grade per $24 \mathrm{~h}$ in the groups of patients who did or did not request a dose escalation from 5 - to 10 -mg solifenacin at 4, 12, and 24 weeks (Mann-Whitney test). UUI, urgency urinary incontinence.

or to request a dose increase based on their satisfaction with treatment efficacy and tolerability and discussion with the investigator. These previous studies did not compare between nonescalators and escalator but rather between a placebo group and a drug administration group (including 5- or 10-mg solifenacin). Recently, the SUNRISE study investigated the relationship between the severity of baseline OAB symptoms and requests for a solifenacin dose increase and the efficacy of 5- and 10 -mg solifenacin doses in relieving OAB symptoms in patients who requested a dose increase [14]. In the 16-week, prospective, double-blind, double-randomized, parallel-group, multicenter study, 591 patients received 5- or 10-mg solifenacin once daily in a flexible-dose regimen. The present study examined the differences between escalators and non-escalators (DE group vs. NE group) using a flexible-dose solifenacin regimen with a 3-day voiding diary, $\mathrm{OAB}$ questionnaires, and uroflowmetry for 24 weeks in a real clinical setting [3].

The patients who chose $\mathrm{DE}$ tended to have more severe $\mathrm{OAB}$ symptoms at baseline; this was consistent across all the variables in $\mathrm{NE}$ versus $\mathrm{DE}$. In the present study, $\mathrm{OAB}$ wet, all diary variables, and total OABSS were higher in baseline characteristics between the 5- and 10-mg solifenacin groups. Serati et al. [15] evaluate to identify the factors associated with the risk of solife- nacin treatment failure. Specifically, the daily number of episodes of urgency, and the different symptoms severity scores (overactive bladder questionnaire-short form and USS) could be considered predictors of solifenacin failure. For these patients, an increase of the solifenacin dosage might be useful. Chapple et al. [13] reviewed data from several studies with solifenacin, darifenacin and oxybutynin extended-release that evaluated the impact of dose flexibility on clinical management. This study reported that patients with OAB refractory to initial treatment who requested dose increase, had often more severe symptoms at inclusion, especially in terms of number of episodes of urgency (with or without urge incontinence)

Large meta-analysis trials, including the STAR, SUNRISE, and VENUS studies, performed with this drug clearly demonstrated significant improvements in $\mathrm{OAB}$ symptoms compared with the placebo [16]. The drug effects were similar regardless of solifenacin dose ( 5 and $10 \mathrm{mg}$ ) at the end of the study period [16]. The 5-mg solifenacin group showed a reduction in mean micturitions per day from -0.99 to -1.23 to $-0.75(\mathrm{P}<0.01)$ compared with the 10-mg solifenacin group ( -1.3 to -1.56 to $-1.04, \mathrm{P}<0.01)$. The mean values of urgency episodes and UUI episodes per day showed similar patterns in both groups. In contrast, our results indicate that improvements in mean epi- 
sodes were maintained or further increased in the DE group compared with the NE group after 12 weeks (patients had a second opportunity for DE). A difference was observed in the results of the two groups caused by escalating the dose twice in the DE group.

Regardless of the dose escalator groups having lower drug efficacy before $\mathrm{DE}$, a gradual reduction in the differences in $\mathrm{OAB}$ symptoms between the two groups was evident. By week 4 , the mean percentage improvements in diary variables were significantly smaller in the DE group than in the NE group (all $\mathrm{P}<0.05)$. Drug efficacy in the $\mathrm{DE}$ group became similar to that in the NE group. At week 12, the number of UUI episodes per 24 hours was the only variable that was statistically different between the two groups. At the end of the study, no statistical differences were observed between the groups for any diary variables.

It is difficult to compare our results directly with flexible-dose studies of other anticholinergics. In a fesoterodine study, at week 4 (before the decision to escalate), all the outcomes were significantly improved from the baseline in both groups (all $\mathrm{P}<0.05$ ), and no significant differences were observed between the nonescalators and escalators at week 12, except UUI episodes per 24 hours $(\mathrm{P}<0.05)$ [17]. The fesoterodine study also showed similarities with our results in that the number of UUI episodes was the slowest variable to respond to the anticholinergic drug. A dose increase was requested by $41.2 \%$ of patients receiving 5 -mg solifenacin after 4 or 12 weeks of active treatment. The incidence of DE in our study is similar to that in previous reports; a dose increase was requested by $48 \%$ of patients on solifenacin in the STAR study [8], 47\% in the SUNRISE study [6], and 44.1\% in the VENUS study [9].

This study had several limitations. It had a retrospective design, and only patients who continued the drug for 24 weeks were included, which is inherently a selection bias (all the patients who discontinued or did not comply for 24 weeks are not included in this population). There was great opportunity for bias in this small population, although the patients were followed up according to the protocol of our department. Objective parameters, including micturition, urgency episode, and urgency incontinence episode data, were used to make an objective comparison between dose escalators and nonescalators. However, a subjective measurement index of changes in urgency incontinence, treatment satisfaction, and quality of life was lacking.

In conclusion, the flexible-dose treatment with solifenacin improved $\mathrm{OAB}$ symptoms in the patients who chose to main- tain the initial dose, as well as in $41 \%$ of those who chose to escalate the dose after 4 or 12 weeks. Although the reduction in $\mathrm{OAB}$ symptoms was insufficient in the $\mathrm{DE}$ group before $\mathrm{DE}$, the gap narrowed steadily to be similar by the end of the study period (24 weeks). Patients with unfavorable initial symptoms had a tendency to escalate to $10-\mathrm{mg}$ solifenacin and showed significant improvements in $\mathrm{OAB}$ symptoms. The use of flexible-dose solifenacin ( 5 or $10 \mathrm{mg}$ ) is associated with UUI and higher total OABSS.

\section{CONFLICT OF INTEREST}

No potential conflict of interest relevant to this article was reported.

\section{REFERENCES}

1. Abrams P, Cardozo L, Fall M, Griffiths D, Rosier P, Ulmsten U, et al. The standardisation of terminology of lower urinary tract function: report from the Standardisation Sub-committee of the International Continence Society. Neurourol Urodyn 2002;21:167-78.

2. Lee YS, Lee KS, Jung JH, Han DH, Oh SJ, Seo JT, et al. Prevalence of overactive bladder, urinary incontinence, and lower urinary tract symptoms: results of Korean EPIC study. World J Urol 2011;29: 185-90.

3. Irwin DE, Milsom I, Kopp Z, Abrams P, Artibani W, Herschorn S. Prevalence, severity, and symptom bother of lower urinary tract symptoms among men in the EPIC study: impact of overactive bladder. Eur Urol 2009;56:14-20.

4. Liberman JN, Hunt TL, Stewart WF, Wein A, Zhou Z, Herzog AR, et al. Health-related quality of life among adults with symptoms of overactive bladder: results from a U.S. community-based survey. Urology 2001;57:1044-50.

5. MacDiarmid SA. Maximizing anticholinergic therapy for overactive bladder: has the ceiling been reached? BJU Int 2007;99 Suppl 3:8-12.

6. Cardozo L, Hessdorfer E, Milani R, Arano P, Dewilde L, Slack M, et al. Solifenacin in the treatment of urgency and other symptoms of overactive bladder: results from a randomized, double-blind, placebo-controlled, rising-dose trial. BJU Int 2008;102:1120-7.

7. Abrams P, Swift S. Solifenacin is effective for the treatment of $O A B$ dry patients: a pooled analysis. Eur Urol 2005;48:483-7.

8. Chapple CR, Martinez-Garcia R, Selvaggi L, Toozs-Hobson P, Warnack W, Drogendijk T, et al. A comparison of the efficacy and tolerability of solifenacin succinate and extended release tolterodine 
at treating overactive bladder syndrome: results of the STAR trial. Eur Urol 2005;48:464-70.

9. Karram MM, Toglia MR, Serels SR, Andoh M, Fakhoury A, Forero-Schwanhaeuser S. Treatment with solifenacin increases warning time and improves symptoms of overactive bladder: results from VENUS, a randomized, double-blind, placebo-controlled trial. Urology 2009;73:14-8.

10. Jeong SJ, Homma Y, Oh SJ. Korean version of the overactive bladder symptom score questionnaire: translation and linguistic validation. Int Neurourol J 2011;15:135-42.

11. Coyne KS, Tubaro A, Brubaker L, Bavendam T. Development and validation of patient-reported outcomes measures for overactive bladder: a review of concepts. Urology 2006;68(2 Suppl):9-16.

12. Andersson KE. Antimuscarinics for treatment of overactive bladder. Lancet Neurol 2004;3:46-53.

13. Chapple CR, Rosenberg MT, Brenes FJ. Listening to the patient: a flexible approach to the use of antimuscarinic agents in overactive bladder syndrome. BJU Int 2009;104:960-7.

14. Cardozo L, Amarenco G, Pushkar D, Mikulas J, Drogendijk T, Wright $\mathrm{M}$, et al. Severity of overactive bladder symptoms and response to dose escalation in a randomized, double-blind trial of solifenacin (SUNRISE). BJU Int 2013;111:804-10.

15. Serati M, Braga A, Siesto G, Sorice P, Cattoni E, Uccella S, et al. Risk factors for the failure of antimuscarinic treatment with solifenacin in women with overactive bladder. Urology 2013;82:1044-8.

16. Chapple CR, Khullar V, Gabriel Z, Muston D, Bitoun CE, Weinstein D. The effects of antimuscarinic treatments in overactive bladder: an update of a systematic review and meta-analysis. Eur Urol 2008; 54:543-62.

17. Wyndaele JJ, Goldfischer ER, Morrow JD, Gong J, Tseng LJ, Choo MS. Patient-optimized doses of fesoterodine improve bladder symptoms in an open-label, flexible-dose study. BJU Int 2011;107: 603-11. 